\title{
LA CANCIÓN POPULAR COMO FUNDAMENTO DEL APRENDIZAJE MUSICAL: EL CASO DE MALLORCA ${ }^{1}$
}

\author{
Arnau Reynés Florit ${ }^{2}$ \\ Llorenç Gelabert Gual ${ }^{3}$
}

\begin{abstract}
Great music educators of the twentieth century have not hesitated to take popular music and songs as the starting point for musical education in children. Hungarian Zoltan Kodály, a great supporter of this proposal, has pointed out how easily children assimilate music which is part of their own culture, of what they have heard and lived in their own environment. Without wishing to delve too much in Kodály's ideas, which have been widely studied, we shall analyze and discuss the contributions of three Majorcan musicians of the present day: Bernat Julià, Antoni Martorell and Baltasar Bibiloni. All three have used popular Majorcan songs as the basis for their compositions at some point in their respective careers. While the first two introduced the popular element into their musical adaptations for elementary and intermediate levels of piano study, Bibiloni incorporated the popular component into his compositions for schools, in vocal arrangements for one, two, or three voices, for voices and Orff instruments, and for voices and piano. In this article we examine and analyze the contributions of these three musicians whose homeland is Mallorca.
\end{abstract}

Keywords: music education; musical pedagogy; popular song; Bernat Julià; Antoni Martorell; Baltasar Bibiloni

Resumen: Grandes pedagogos musicales del siglo XX no han dudado en hacer de la música y canción tradicionales o populares el punto de partida de la enseñanza musical en los más pequeños. El húngaro Zoltan Kodály, como máximo exponente de esos postulados, argumenta la fácil asimilación del hecho musical por parte del niño a partir de aquello que le es más propio, aquello que ha oído y vivido en su entorno. Sin querer profundizar en demasía en las ideas de Kodály, tema ampliamente estudiado, analizaremos las aportaciones de tres figuras musicales de la Mallorca contemporánea: Bernat Julià, Antoni Martorell i Baltasar Bibiloni. Los tres coinciden en el uso de la canción popular mallorquina como base de sus composiciones en algún momento de sus respectivas trayectorias. Si bien los dos primeros enfocaron ese elemento a la composición de adaptaciones de nivel elemental y medio para el estudio del piano, Bibiloni lo trasladó al ámbito escolar en forma de arreglos vocales a una, dos o tres voces, para voces e instrumental Orff o para voces y piano, etc. Con este artículo pretendemos analizar y sacar a la luz todas esas aportaciones desde la realidad que les unió a los tres, su tierra natal, Mallorca.

Palabras clave: enseñanza musical; pedagogía musical; canción popular; Bernat Julià; Antoni Martorell; Baltasar Bibiloni

Reynés Florit, A.; Gelabert Gual, L. (2012). La canción popular como fundamento del aprendizaje musical: el caso de Mallorca. DEDiCA. REVISTA DE EDUCAÇÃO E HUMANIDADES, 3 (2012) março, 287-302 


\section{Introducción}

El uso de la canción popular o tradicional como eje pedagógico de la enseñanza musical podemos visualizarlo en las teorías y métodos de los grandes pedagogos europeos del siglo XX. El método Orff-Schulwerk, por ejemplo, enfatiza el ritmo intrínseco de dichos, refranes y canciones populares, adaptándolos a la enseñanza de la música en los más pequeños (Sanuy; González, 1969). El catalán Joan Llongueres, discípulo directo del suizo Émile Jaques-Dalcroze(1865-1950), hará una adaptación de su Rítmica a partir del uso de la canción y danza popular catalanas (Llongueres, 1923; Llongueres 1942), entendidas estas como elementos más cercanos y naturales al niño, como eje de la enseñanza músical. Zoltán Kodály(1882-1967) destacó por su labor de situar a la canción popular húngara como eje de su método de enseñanza musical. Ese postulado servirá como modelo de estudiosos más cercanos a nosotros en esta materia, como la del catalán Ireneu Segarra (1917-2005) o el propio Baltasar Bibiloni i Llabrés(1936- ). La aportación de Ireneu Segarra significó el punto de partida de una nueva forma de entender la pedagogía musical (Casals, 1993). Su influencia ha perdurado hasta nuestros días, traspasando incluso las fronteras de Catalunya. En el segundo apartado de este artículo analizaremos precisamente la aportación de Bibiloni, muy en connivencia con el ideario de Segarra. Amigo personal del pedagogo catalán trabajó durante años en la difusión de su método a través de la Escola de Pegagogia Musical - Mètode Ireneu Segarra de Catalunya.

Nuestro objeto de estudio incide precisamente en el uso que hacen de la canción popular y tradicional una serie de autores mallorquines. Tanto el Cançoner de Pedagogia Musical de Baltasar Bibiloni i Llabrés, como Arrels de Bernat Julià i Rosselló(1922- ), o Sempreviva de Antoni Martorell i Miralles(1913-2009) son ejemplos del uso y adaptación de canciones tradicionales y populares de Mallorca a la enseñanza musical. En el caso del primero hablamos de la adaptación de esas canciones a la práctica del canto en la escuela. Tanto Arrels como Sempreviva en cambio, son adaptaciones para el estudio y práctica pianística en un nivel inicial o medio.

Esta aportación, en el caso de Baltasar Bibiloni, tendrá lugar en un contexto social, político y educativo muy determinado. La década de los años setenta del siglo XX coincide con el final de un periodo histórico en España marcado por el estancamiento y la 
negativa a cualquier aportación pedagógica 0 metodológica procedente de la vanguardia europea. El ámbito de la educación musical no vivirá ajeno a esos aires de cambio. La música empieza a asumir una cierta presencia en los centros educativos y los conservatorios empiezan a ver alternativas a sus métodos anacrónicos. Será hacia esos ámbitos a los que los que tanto Bibiloni, como Julià i Martorell posteriormente, aportarán sus iniciativas. Precisamente por la influencia de los grandes metodistas musicales del siglo XX y por la tendencia creativa de los grandes compositores, la música popular y tradicional asumirá un papel relevante en esas composiciones. Tan relevante en el caso de Bibiloni que gran parte de su obra se basa en arreglos y adaptaciones de música tradicional de Mallorca y de las Islas Baleares.

Efectivamente, el hecho de hacer uso de este elemento tan propio y cercano como es la canción popular y tradicional, y situarlo como punto de partida de todo un engranaje pedagógico o compositivo, no es fruto del azar. Este hecho vendrá marcado por un trasfondo de reivindicación de lo propio ligado a aspectos sociales y políticos que no procede analizar en este artículo. Sin embargo consideramos oportuno establecer un marco histórico y legislativo previo al análisis de las aportaciones de los tres autores, marco en el cual los tres desarrollaron su legado.

\section{Enseñanza e instituciones musicales en Mallorca}

Coincidiremos todos en que la educación general, tal y como la entendemos hoy en día después de más de un siglo de escuela obligatoria en España, tiene como meta ineludible, entre otras, el hecho de contribuir y ayudar a desarrollar la inteligencia y sensibilidad del individuo. En el caso de la música que nos ocupa, el mensaje estético que esta desprende va íntimamente ligado al ámbito de los sentimientos. Podemos concluir entonces que el arte musical se convierte en una disciplina que incide directamente en la formación y desarrollo de esta sensibilidad a la que hacíamos referencia. Este razonamiento, que a muchos nos puede parecer de lo más obvio y aséptico, no se ha visto reflejado en forma de presencia o reconocimiento reglado de la música en la enseñanza obligatoria hasta hace pocas décadas.

Desde la Ley Moyano de 1857, primer texto legislativo que reconoce la educación como obligatoria para todos los individuos en un umbral determinado de edad, deberá transcurrir más de un siglo para que la música se haga presente de forma reglada dentro la 
escuela (ver Artículo 47 del Título III de la Sección Primera de la Ley Moyano, donde encontramos una referencia a los estudios de Bellas Artes, entre ellos la música, incluidos estos en el grupo de Enseñanzas Superiores).

La aprobación de la Ley General de Educación de 1970 implicará un cambio notable en este aspecto. La música alcanza un espacio dentro de este nuevo texto legislativo (el Artículo 16 de la Ley 14/1970 de 4 de agosto, General de Educación y Financiación de la Reforma Educativa, contempla la "iniciación en la apreciación y expresión estética y artística". Los contenidos en materia de Educación Musical, incluidos estos en el área de Educación Artística, vienen detallados en la Orden de 17 de enero de 1981, BOE núm. 18 de 21/01/1981. En este se regulan las enseñanzas de Educación Preescolar y del ciclo inicial de la Educación General Básica). En el caso de la Educación Primaria, la música no alcanza el estatus de área curricular como tal. Esta aparece como bloque dentro del área de Educación Artística. Contemplará ya entonces una secuenciación de contenidos nunca vista hasta el momento y que, en cierto modo, debe contribuir a la consolidación y normalización de la enseñanza musical en las escuelas. Dicha normalización nunca se llevó a la práctica debido a la falta de profesorado especializado en la materia.

La LOGSE de 1990 (ver Real Decreto 1006/1991, de 14 de junio, BOE número 152 de 26/6/1991. En este se establecen los objetivos correspondientes al nivel de Educación Primaria en todas las áreas de enseñanza. Entre ellas la Educación Artística, que incluye la expresión musical, plástica y dramatización) y la LOE del 2006, últimas leyes de educación aprobadas e implantadas en España, no han introducido cambios significativos en cuanto a la presencia de la música en la enseñanza primaria. Si bien la LOGSE elabora un plan de estudios riguroso en cuanto a contenidos a impartir y objetivos a alcanzar así como la metodología a utilizar, no contempla en ningún caso un aumento en presencia lectiva de Educación Musical.

Si bien no hemos visualizado cambios significativos en cuanto a presencia de la música en la escuela obligatoria desde la década de los setenta del siglo XX, no podemos decir lo mismo por lo que se refiere a la regulación de los estudios musicales en los conservatorios. Precisamente la LOGSE, a través de las llamadas Enseñanzas de Régimen Especial, favorecerá i facilitará el hecho de compaginar estudios musicales de conservatorio con la enseñanza 
obligatoria, tanto en el grado elemental como en el grado medio, homologando incluso el título de bachiller una vez superadas las materias comunes de esta etapa educativa. La superación de los estudios superiores se equipara, a todos los efectos, al título de Licenciado Universitario (en los Artículos 39, 40, 41 y 42, Capítulo Primero del Título Segundo de las Enseñanzas de Régimen Especial de la Ley Orgánica General del Sistema Educativo de 3 de octubre de 1990, BOE de 4 de octubre, podemos consultar las referencias explícitas que contempla esta ley referidas a la enseñanza de estudios musicales).

Acabamos de visualizar cómo la enseñanza musical reglada en España viene dada desde dos ámbitos formativos y dirigidos a un perfil de alumno diferente. En primer lugar nos referimos al ámbito de la educación general, donde la labor de los profesionales que impartirán música tendrá como premisa básica la formación cívica e intelectual de personas a través de la música. La formación de estos profesionales tiene lugar en el ámbito universitario a través de los estudios de maestro o pedagogía musical. En segundo término hemos hablado de los conservatorios: instituciones dedicadas eminentemente a la formación de futuros músicos profesionales. Seguidamente haremos un repaso de las instituciones de enseñanza musical que han cubierto ambas vertientes de enseñanza en las Islas Baleares.

En el ámbito de la formación de futuros docentes de la educación general, destacamos una primera escuela de maestros en Mallorca datada de 1842: el Seminario de Maestros, llamada posteriormente Escuela Normal Masculina (Mulet, 1987). En esta escuela no se impartía todavía la formación musical. Fue a partir de 1914 cuando se convierte en materia obligada en la formación de todos los maestros y con una duración de dos años. Los contenidos de estos dos cursos de formación musical se basaban en la lectura musical o solfeo y en el aprendizaje de un repertorio de canciones. Antes de 1936 podían incluso iniciarse en el estudio del piano aquellos alumnos que lo desearan. No obstante, esta dedicación no era suficiente para preparar a maestros con una formación pedagógica adecuada en música. En 1971 se da un paso atrás cuando los estudios de maestro se convierten en diplomaturas. La música pierde tiempo de dedicación en todas las especialidades (Ciencias Sociales, Filología y Ciencias), pasando de dos cursos a uno, salvo la especialidad de Preescolar donde se mantienen los dos. En el caso de la Escuela Normal de Formación del Profesorado 
de Palma de Mallorca se pudo incluir una asignatura optativa de música poco tiempo después (Janer, 2004). Con la LOGSE de 1990 se produce un hecho capital creándose la especialidad de los estudios de Maestro en Educación Musical. Esta nueva especialidad contempla una serie de asignaturas que abarcan desde la formación en el lenguaje musical hasta la formación en didáctica musical. En el curso universitario 2009-10 y en el nuevo marco del Espacio Europeo de Estudios Superiores implantado en la Universidad de las Islas Baleares, desaparecerá la especialidad en Educación Musical. De las siete especialidades que contemplaba la LOGSE pasaremos a dos de carácter más generalista: Maestro en Educación Primaria y Maestro en Educación Infantil. Estas dos especialidades incluirán materias dedicadas a lo que anteriormente eran las especialidades, ahora llamadas menciones, aunque con una presencia mucho menor.El que a día de hoy es el Conservatorio Profesional de Música y Danza de Palma de Mallorca, se fundó en el año 1935 como Conservatorio Regional de Baleares. Destacó en sus inicios por la enorme calidad pedagógica y artística de su profesorado. Personalidades de prestigio como Joan María Thomàs, Jaume Mas Porcel, Ignacio Piña, Teresa Bover o Rafael Vich fueron algunos de los músicos que impartieron docencia en los inicios. Este conservatorio siguió las directrices y modelo del Conservatorio de Madrid de entonces, impartiendo las llamadas enseñanzas superiores instrumentales. Esta brillante etapa se vio interrumpida de nuevo por una resolución de la Dirección General de Bellas Artes de 1942, que rebajaba la categoría del Conservatorio Profesional a Elemental. No será hasta el año 1977 cuando recupere el estatus de Profesional, en el marco de la Ley General de Educación de 1970. En el año 2001 se visualizará la presencia de estudios superiores de música en las Islas Baleares con la creación del Conservatorio Superior de Música de las Islas Baleares (Bibiloni; Moll; Roig et al., 2004).

Hablamos a día de hoy entonces, de dos grandes instituciones de formación musical en las Islas Baleares: los conservatorios, en sus tres modalidades (elemental, profesional y superior) y la Universidad de las Islas Baleares, encargada de formar a futuros maestros que impartirán música en centros escolares. Será precisamente a los alumnos de estas instituciones musicales a quienes Baltasar Bibiloni, Bernat Julià y Antoni Martorell dedicarán parte de su obra pedagógica, que analizaremos seguidamente. Lo harán además en una coyuntura de efervescencia 
del hecho musical en general y de lo autóctono en particular, tanto en la vertiente educativa como compositiva. Este proceso de apertura que se visualiza de facto en el ámbito escolar en la década de los setenta del siglo $\mathrm{XX}$, se vislumbrará también en los conservatorios pasados unos años. En el caso de Mallorca se empezará a introducir repertorio de canciones tradicionales adaptadas al estudio del piano y compuestas por autores mallorquines a principios de la década de los noventa. Julià y Martorell serán sus máximos exponentes.

\section{Aplicación y presencia de la canción popular en la enseñanza musical de Mallorca}

\section{1. La canción popular de Mallorca}

Cuando hablamos de canción popular o tradicional nos referimos a aquellas melodías que han sido asimiladas y apropiadas por el propio pueblo, convirtiéndose en parte importante de su idiosincrasia cultural y folklórica. Otra característica generalizable de la canción popular es su autoría, en la inmensa mayoría de casos anónima. Este anonimato se debe a la longevidad de dichas canciones y al hecho de tratarse generalmente de melodías sencillas que se han ido transmitiendo de forma oral y de generación en generación, siendo estas susceptibles a cambios con el paso del tiempo. Sin embargo, hay casos excepcionales de canciones que se han convertido en populares aun conociéndose su autoría. S'estrella de s'auba, con letra de Marià Aguiló y música de Miquel Tortell, sería una ejemplo de canción mallorquina de origen culto convertida después en tradicional y popular. Otro ejemplo seria Vou, veri vou, canción de cuna del también compositor mallorquín Honorato Noguera. Ambas canciones forman parte del patrimonio artístico musical Mallorca, y se han convertido en parte viva del espíritu popular mallorquín.

Felip Pedrell en su Cancionero Musical Español (Pedrell, 1922) define el canto como un lenguaje capaz de expresar los sentimientos del hombre. Prosigue definiendo al canto que ha nacido del pueblo y que ha permanecido en pie a lo largo de los siglos y es reflejo del propio pueblo, como canción popular. El etnomusicólogo catalán Josep Crivillé i Bargalló nos habla del efecto terapéutico del canto popular, de su simbolismo, su magia, y hasta de su poder curativo (Crivillé, 1983).

En el ámbito religioso y litúrgico también se han popularizado melodías de autor. El Cantoral Religioso de Bernat 
Julià i Rosselló es un ejemplo (Julià, 1955). Escrito en lengua catalana, se difundió por todas las parroquias de las Islas Baleares. Posteriormente Antoni Martorell i Miralles crea un cancionero religioso también en lengua catalana e inspirado en la música popular mallorquina (Martorell, 1990). Este cancionero religioso se ha utilizado en la mayoría de parroquias mallorquinas, convirtiendo sus melodías en cantos populares de la liturgia mallorquina.

Caben destacar las recopilaciones de canciones populares y tradicionales de Mallorca llevadas a cabo desde la década de los años ochenta del siglo XX por Josep Massot i Planas (Massot, 1984) o Miquel Julià i Prohens (Julià, 1969). Estas recopilaciones fueron recogidas a partir de testimonios orales y posteriormente adaptadas y editadas en partitura. Dichas ediciones son referencia y configuran gran parte del patrimonio del canto popular y tradicional de Mallorca.

\section{2. Aplicación a la enseñanza}

En las últimas dos décadas ha sido frecuente la presencia de la canción popular y tradicional en lengua catalana en los libros de texto de música de Educación Primaria. Este hecho se da de manera generalizada en Cataluña y las Islas Baleares, donde las editoriales se han preocupado de hacer uso de las canciones populares propias de cada lugar dentro de las propuestas didácticas que incluyen sus publicaciones. Esta nueva tendencia venía precedida de una concepción mucho más etnocentrista de la enseñanza donde sólo la música de tradición culta podía tener cabida.

A nivel pedagógico, esta revalorización de lo más sencillo y cercano a nuestro medio se presenta como un elemento facilitador del proceso de enseñanza y aprendizaje de la música. Este postulado tuvo como principal avalista e impulsor al húngaro Zoltán Kodály, quien a través de una exhaustiva investigación del cancionero y folklore popular húngaro, creó entonces toda una metodología de enseñanza musical vertebrada a partir de la canción popular. Carl Orff (1895-1982) también hizo uso de los elementos folclóricos de su país para la elaboración de su método. El ejemplo más cercano a Bibiloni de esta tendencia es el del catalán Ireneu Segarra, monje del Monasterio de Montserrat deslumbrado y inspirado por la idea de Kodály. Este último elaboró un método de nueva creación dirigido a la formación musical en las escuelas catalanas. La base metodológica empleada por Ireneu Segarra se acoge a un modelo basado en la pentatonía en los primeros dos cursos, seguido de la diatonía, la fonomimia y el solfeo relativo, con 
la canción popular catalana de trasfondo. No obstante, y más allá de estas premisas iniciales, el objetivo principal según Segarra es hacer de la música una expresión de vida y comunicación a nivel artístico. Por eso, el catalán destaca como capital el hecho vivencial que lleva implícita la música, más allá de todos los aspectos de carácter teórico y que obviamente han y debe complementar y dar sentido y forma a la vivencia.

El hecho de que en la canción popular catalana no esté presente el pentatonismo, que sí lo estaba en el folklore húngaro y significaba la base del método de Kodály (en su método estas canciones pentatónicas aparecen secuenciadas de menor a mayor dificultad), obligó en Segarra a crear una serie de melodías que facilitaran la incorporación escalonada de nuevos elementos musicales. Baltasar Bibiloni, que trabajó con Segarra en la Escuela de Pedagogía Musical de Cataluña donde se venía aplicando su método, fue el introductor de estas ideas a la realidad de las Islas Baleares. Lo hizo a través de su práctica docente tanto en los estudios de magisterio como en los cursos de verano de la Universidad de las Islas Baleares. La importación de estas ideas vino acompañada, como ya hemos mencionado en puntos anteriores, de la creación de una serie de arreglos y adaptaciones de melodías populares de las Islas Baleares. Se basó precisamente en melodías populares editadas en las recopilaciones de Josep Massot i Muntaner i Miquel Julià i Prohens, citados anteriormente.

En el caso de Arrels i Sempreviva, de Bernat Julià i Antoni Martorell respectivamente, se hará uso también de estas mismas canciones populares pero adaptadas al estudio del piano en niveles iniciales. A diferencia de Bibiloni, más centrado en la línea puramente pedagógica de las vanguardias europeas, el uso de la canción popular por parte de Julià i Martorell obedece más a un componente de reivindicación patriótica o nacionalista. Si bien la interpretación de dichas composiciones se desarrollará principalmente en los conservatorios.

\section{3. Baltasar Bibiloni. Aplicación a la pedagogía musical y su didáctica}

Empezar a aprender música haciendo música, y hacerlo a partir de aquella que nos es más cercana, era algo que Baltasar Bibiloni ya había intuido y pensado antes de conocer las propuestas de Kodály o Segarra. El hecho de toparse con unas metodologías tan conniventes con lo que su instinto le dictaba, despertó en él la necesidad de acercarse y profundizar. Fruto de esas influencias y a 
partir de su experiencia docente, estableció una hoja de ruta en su labor pedagógica (Bibiloni, 1984). Dicha hoja de ruta se basa en diferentes aspectos que giran en torno a un eje de aprendizaje, la canción popular. Son los siguientes:

- La canción: Baltasar Bibiloni considera a la canción como el eje de la enseñanza de la música en la escuela. Se basará en la canción autóctona y popular de las Illes Balears y tierras de habla catalana en general. Considerará capital el aprendizaje de un repertorio básico de canciones adaptadas a los distintos niveles de enseñanza.

- Educación de la voz: Este parámetro de enseñanza, según Bibiloni, va muy ligado a la canción. Es más, a través de esta se profundiza y se hace incidencia en aspectos de técnica vocal a través de ejercicios adaptados a los niños.

- Audición: Es un complemento indispensable al resto de parámetros, según Bibiloni. Todo ello a partir del acercamiento de la música de los grandes maestros a los niños, y a partir de ella ir descubriendo elementos técnicos trabajados o por trabajar. Fomentará la audición en directo.

- Educación del oído: El trabajo de este aspecto resulta fundamental e indispensable de cara a una completa formación musical de los niños, según Bibiloni. Reconoce que la fijación de las relaciones interválicas es el aspecto de maduración más lento en el proceso de formación, ya que es el más abstracto. A menudo olvidada, una buena educación del oído es la base sólida de los futuros músicos. Sin ésta no podemos hablar de educación musical.

- Ritmo-movimiento: Indisoluble del trabajo de oído que hemos mencionado antes, Bibiloni incluye paralelamente el trabajo sensorial del ritmo. Basado en los estadios iniciales en una educación motriz a muchos niveles, musicalmente hablando se basará en la pulsación.

- Lectura y escritura: En referencia a este aspecto, Bibiloni hace suyas las siguientes palabras de Kodály, donde reflexiona sobre la importancia de la alfabetización musical

La vertiente compositiva de Baltasar Bibiloni ha ido ampliando sus horizontes con el paso del tiempo. Lo que en principio se centraba en la creación de arreglos sencillos de cara a su labor docente, se ha convertido en una auténtica vocación a día de hoy. Con un legado de más de ciento cincuenta obras compuestas, todas ellas destacan por un elemento común: la 
presencia del elemento vocal, ya sea para agrupación coral o para solistas.

A pesar de un amplio abanico de composiciones hechas a partir de textos de autores catalanes, en este artículo hemos querido centrarnos exclusivamente en la presencia y arreglos de la música popular en lengua catalana. "Sentía envidia por el hecho que en Cataluña tuvieran un abanico amplísimo de canciones tradicionales arregladas para la práctica del canto. En Mallorca no existía entonces material editado de este tipo. Entonces decidí ponerme a trabajar en este sentido. La primera canción de la cual hice un arreglo para coro mixto fue el popular Copeo Matancer. A partir de ésta, hice toda una serie de armonizaciones de canciones populares de las Islas Baleares. Cogía muchas melodías del libro de canciones populares de Miquel Julià». Así de contundente se manifestaba Bibiloni de regreso a Mallorca, después de permanecer dos años en Barcelona formándose en armonía y composición.

Seguidamente haremos una clasificación y un pequeño comentario de todos sus arreglos de canciones populares, publicadas o no. Elaborados en principio para su práctica docente cotidiana y para ser cantados por las corales que dirigía entonces, con el paso de los años se han convertido en un material de uso generalizado por parte del colectivo de maestros de música en todas las Islas Baleares, así como los directores de corales de tierras de habla catalana. Al título de la obra o edición le seguirán la agrupación por la que debía ser interpretada (coro mixto, coro a dos voces blancas, coro a tres voces blancas y piano, etc) y la procedencia de la canción o canciones populares:

- Cançó de l'absència(1982): Coro-4 voces mixtas. Tradicional mallorquina. Inicio con melodía soprano y boca cerrada el resto de voces.

- Anàrem a Sant Miquel(1989): Coro-4 voces mixtas. Tradicional ibicenca.

- Com voleu germans que canti(1989): Coro-4 voces mixtas. Tradicional ibicenca.

- Cançó de la núvia impacient(1998): Voz y piano. Tradicional mallorquina. Composición para voz y acompañamiento de piano.

- Cinc cançons mallorquines(1970-80): Coro-4 voces mixtas. Cançó de la neu: villancico; Copeo matancer: copeo mallorquín; Bressol de Nadal: villancico para solista y 4 voces mixtas; Una dona llarga $i$ prima; El taronger. 
- Cançoner de pedagogia musical(1970-80): Arreglos sobre canciones y refranes de Mallorca. Tradicionales mallorquinas. 3 arreglos para voz y acompañamiento rítmico, de melodía y ritmos muy básicos y asequibles para Educación Primaria, 8 para voz e instrumental Orff de dificultad media (último ciclo Primaria, Secundaria o Escuelas de Música), 13 para 2 voces blancas (coros infantiles y juveniles de nivel medio), y finalmente rítmica prosódica, asequible a cualquier nivel.

- Cançons per a Instrumental Orff(1970-80): 24 arreglos de canciones tradicionales mallorquinas, catalanas, valencianas, sudamericanas, norteamericanas, europeas, una vasca y una israelí, para instrumental Orff, voz y flauta en alguna de ellas. Material útil para Educación Primaria, Secundaria y Escuelas de Música.

- 20 cançons tradicionals per a veus blanques i piano(1970-80): 20 arreglos de canciones tradicionales para 1, 2 o 3 voces blancas y piano. Tradicionales de tierras de habla catalana. El hecho de que la mayoría sean a 2 o 3 voces hace que sea un material dirigido a un coro infantil o juvenil con cierta experiencia. Muy adecuado para Escuelas de Música.

- La dama de Mallorca(1982): Coro-4 voces mixtas. Tradicional mallorquina.

- Dues cançons populars menorquines: Na Falariareta i Sa vella Rabisca (1984): Coro-2 y 3 voces blancas. Tradicionales menorquinas. Na Falariareta: armonización para 3 voces blancas. Adecuada para coros infantiles y juveniles; Sa vella Rabisca: armonización para 2 voces blancas. Adecuada para coros infantiles y juveniles.

- Ton pare no té nas(1987): Coro-4 voces mixtas. Tradicional mallorquina.

- Rosa Vera(1989): Coro-4 voces mixtas. Tradicional ibicenca.

- Cuatre cançons tradicionals(1993-2001): Coro-3 voces blancas, solista y piano. Tradicionales de tierras de habla catalana. Adecuados para coros juveniles y de voces blancas con cierto nivel musical debido a las dificultades rítmicas y melódicas que aparecen. Incluye: Garrideta, Parado de Valldemossa, El bon Jesuset, Ton pare no té nas.

\section{4. Bernat Julià y Antoni Martorell. Aplicación didáctica al piano y al órgano}

El gran pedagogo musical y compositor húngaro Bela Bartok(1881-1945) revolucionó el panorama musical en cuanto a la 
didáctica del piano. Su obra Mikrokosmos, que consta de 6 volúmenes y 156 composiciones, supone una de las aportaciones más importantes en la historia de la pedagogía pianística. Esta obra apuesta por una nueva metodología de características claramente definidas. Entre ellas destacan el uso de la música folklórica húngara como elemento de partida, la improvisación y la práctica vocal, el uso de material a cuatro manos (útil para dos alumnos o alumno y profesor), arreglos de corta duración, uso de títulos propios referidos al aspecto técnico a tratar, aparición de nuevos conceptos armónicos y rítmicos, adaptaciones a todos los niveles, etc. Tanto Bernat Julià como Antoni Martorell se sumaron al modelo de Bartok, adaptando la experiencia del gran músico húngaro a las raíces musicales y folklóricas de Mallorca.

Arrels (Julià, 1989) de Bernat Julià i Sempreviva de Antoni Martorell (Martorell, 1992) serían las obras pianísticas de ambos compositores con un componente pedagógico más visible. Dirigidas a intérpretes de grado elemental y medio, constituyen ambas un compendio de arreglos de música popular de Mallorca adaptadas a la práctica pianística. Ambas coinciden en su objetivo didáctico: formar a los alumnos de las escuelas de música y conservatorios en sus inicios en el estudio del piano. $Y$ lo hacen precisamente a través de un elemento cercano a esos alumnos, la canción popular y tradicional de Mallorca.

Al componente educativo de las dos obras anteriormente mencionadas cabe añadir el hecho de introducir la canción popular al ámbito académico y posibilitar y facilitar con ello su prestigio y difusión. Además de fortalecer la difusión del gran patrimonio de la canción popular de Mallorca, ambos autores nos presentan una nueva forma de amarlo y disfrutarlo a través del instrumento de tecla por excelencia, el piano. En la edición de Sempreviva, Martorell dedica unas palabras a los intérpretes a los cuales van dedicadas estas armonizaciones: "A los niños y niñas, candidatos a pianistas, del Conservatorio de Música de las Islas Baleares candidatos a pianistas, alentándoles a conocer y amar nuestro folklore musical".

En Arrels y Sempreviva aparecen diferentes parámetros técnicos a trabajar por parte del estudiante: la digitación, el uso del pedal y la articulación. Antoni Martorell añade además en Sempreviva un apéndice que trata la referencia modal. Estas obras de Julià y Martorell, de carácter eminentemente pedagógico, coinciden en una serie de objetivos transversales que nos conducen a la concepción de un aprendizaje global. Estos objetivos van desde 
los puramente técnicos y mecánicos mencionados, hasta otros más globales de índole artística y cultural. A través de estos últimos el folklore musical de Mallorca se convierte en un potente activo de intervención educativa.

Las canciones populares o tradicionales que aparecen en Arrels de Bernat Julià son: Sant Joan Pelós; Pep Gonella; Lileta, lilè; Una dona llarga i prima; Cançó dels darrers diez; Com voleu germans que canti; Sa Roqueta; Sant Antoni i el dimoni; Dansa; Cançó de bressol; Bona nit blanca roseta; Un senyor damunt un ruc; El mestre; Monja per força; Plou i fa sol; Sa Ximbomba; Caragol treu banya; Sa serena cau menuda; Madò Catalina; Nai, nai, ja tenc un ventall; Sor Tomasseta; Una taringa de tot es carrer; Na Catalina de plaça; So de pastera.

En Sempreviva de Antoni Martorell aparecen: Pastoret, d'on vens?; A ca s apadrina; Cançó de pastar; El sen Piris; Tonada "des batre metles"; Pipa treu foc; Cançó de festeig; Sa molinereta; La quadrilla des porró; Sant Joan Pelós; Dormirà es meu fillet; A la ciutat de Nàpols; Arri arri somereta; Tonada de picar càrritx; Ball de cavallets; Es molí de'n Jordi Vives; Cançó de's Moliner; Cançó de's segar, Cançó de veremada, Mare de Déu, on anau?, El señor Martí; Sa Morena de Torret; Na Maria-Anna s'estufa; Cançó des missatge; Ton pare no té nas; Tonada de vermar; Garrida, vos sou l'ona; Tonada de treure aigua; La filadora fila fila; Tonada des collir oliva dalt; Deixem lo dol; Cançó des llaurador; En Pep Gonella té; Tonada des batre; Cançó d'espadar; Una taringa de tot es carrer; Entrant dins es poble; Cançó de llaurar; Tonada des tondre; L'any coranta vaig segar; La baldufa.

En la obra de Antoni Martorell siempre aparece presente el elemento popular. En esta misma idea de potenciarlo existe otra obra destacable del autor, Danses populars de les Illes Balears (Martorell, 1998). En este caso se trata de adaptaciones de danzas tradicionales de las Islas Baleares también para el estudio del piano. La danza (exceptuando alguna en especial, como los cossiers), a diferencia de la canción popular mallorquina, tiene las raíces más próximas en el tiempo, en el estilo y en la forma. Este volumen de Antoni Martorell, recoge la mayoría de las danzas más divulgadas en el archipiélago balear. Todas ellas en versión pianística. Estas danzas están agrupadas en diferentes estilos: jota, mateixa, fandango, bolero o copeo, ofreciendo un verdadero muestrario de la danza popular balear. Esta danza popular fue objeto de análisis por parte de Antoni Martorell debido a su fervor popular. La mayoría de 
ellas siguen bailándose en fiestas populares e incluso en actos institucionales.

Una última obra con tintes didácticos compuesta por Antoni Martorell seria l'Organista Celebrante (Martorell, 1996). Antoni Martorell dedicó gran parte de su obra al órgano, instrumento que él interpretaba. Esta obra se inspira en los cantos religiosos populares italianos. El hecho de vivir durante muchos años en Roma le empujó a escribirla. Esta recopilación de cantos viene presentada a modo de preludio, al estilo de los preludios populares de Johann Sebastian Bach. Se trata de una composición de formato original que ofrece al joven organista aprender y conocer el patrimonio popular religioso italiano a través de estos preludios de coral con el fin de perfeccionar la técnica en este instrumento tan complejo y de riqueza tímbrica inigualable.

\section{Conclusiones}

El legado compositivo de los autores estudiados, Baltasar Bibiloni, Bernat Julià i Antoni Martorell, y más concretamente la obra pedagógica elaborada a partir de la canción popular y tradicional de Mallorca, ha supuesto una serie de aportaciones desde diferentes vertientes. En primer lugar, y refiriéndonos a la figura de Baltasar Bibiloni, cabe destacar que sus arreglos, composiciones y metodología adaptadas a la enseñanza musical implicaron en su momento la introducción en las Islas Baleares de una nueva forma de entender la pedagogía musical, prestigiándola y demandando una mayor presencia en las escuelas. Tanto Bernat Julià como Antoni Martorell, años más tarde siguieron un camino similar al de Bibiloni elaborando adaptaciones y arreglos dirigidos al estudio del piano en los conservatorios de las Islas Baleares.

El hecho de llevar a cabo tal empresa educativa, y hacerlo los tres a través de un mismo eje metodológico: la canción popular y tradicional de Mallorca, ha servido para revalorizar el sentido de lo propio con capacidad de formar parte principal de un proceso de aprendizaje, el de la música en los más pequeños en este caso. Finalmente, el uso académico de este elemento popular facilitará su conservación y arraigo.

\section{Bibliografía}

Bibiloni , B. (1984). Lección inaugural del curso académico 1984-85 de la Escuela Universitaria de Formación del Profesorado de E.G.B. Palma de Mallorca..

Bibiloni, M.; Roig et al. (2004). Conservatori, 70 anys d'ensenyament musical. Miscel.lània. Estudis Baleàrics, 80/81 (2004) 7-20. 
Casals, J. (1993). El mètode Ireneu Segarra. Revista Música y Educación, 16 (1993) 51-74.

Crivillé, J. (1983). Historia de la música española, vol. 7, El folklore musical, 81-87. Madrid: Alianza Editorial.

Janer, G. (2004). Les músiques de l'Escola Normal: era en temps de postguerra. Estudis baleàrics, 80/81 (2004) 125-128.

Julià, M. (1969). Mallorca, cançons tradicionals. Barcelona: Hogar del Libro.

Julià, M. (1998) Cançoner tradicional de Mallorca. Palma de Mallorca: Documenta Balear.

Julià, B. (1955). Cantoral Religioso. Palma de Mallorca: Diócesis de Mallorca.

Julià, B. (1989). Arrels. Madrid: Real Musical.

Llongueres, J. (1942). El ritmo en la educación y formación general de la infancia. Barcelona: Editorial Labor. Sección II - Educación.

Claret.

Martorell, A. (1990). Celebrem el Senyor. Barcelona: Editorial

Martorell, A. (1992). Sempreviva: àlbum de cançons populars per al petit pianista. Valencia: Editorial Piles.

Martorell, A. (1996). L'Organista Celebrante. Roma: Editrice Franciscanum.

Martorell, A. (1998). Danses Populars de les Illes Balears: Recull selectiu de les danses populars més generalitzades a les Illes Balears en versió per a piano. Valencia: Editorial Piles.

Massot, J. (1984). Cançoner musical de Mallorca. Corrección musical a cargo de Baltasar Bibiloni i Llabrés i de Josep Massot i Muntaner. Palma de Mallorca: Caixa de Balears "Sa Nostra".

Mulet, B. (1987). L'Escola Normal de Mestres en els seus orígens $i$ el reformisme liberal. Palma de Mallorca: Universitat de les Illes Balears.

Pedrell, F. (1922). Cancionero musical popular español, vol.1, 2-3. Barcelona: Ed. Boileau.

Sanuy, M.; González, L. (1969). Orff-Schulwerk. Música para niños. Madrid: Unión Musical Española.

\footnotetext{
${ }^{1}$ Popular songs as a basis for learning music: the case of Majorca

2 Doctor.

Universitat de les Illes Balears (España).

Email: areynes@gmail.com

${ }^{3}$ Doctor.

Universitat de les Illes Balears (España).

Email: llorens.gelabert@gmail.com
} 